\title{
Nemencha : Société et Langue
}

\section{Malek Boudjellal}

\section{OpenEdition}

Journals

Édition électronique

URL : https://journals.openedition.org/encyclopedieberbere/2713

DOI : 10.4000/encyclopedieberbere. 2713

ISSN : 2262-7197

\section{Éditeur}

Peeters Publishers

\section{Édition imprimée}

Date de publication : 5 octobre 2012

Pagination : $5434-5444$

ISBN : 978-90-429-2718-6

ISSN : 1015-7344

\section{Référence électronique}

Malek Boudjellal, « Nemencha : Société et Langue », Encyclopédie berbère [En ligne], 34 | 2012, document N39, mis en ligne le 15 décembre 2020, consulté le 17 février 2022. URL : http:// journals.openedition.org/encyclopedieberbere/2713; DOI : https://doi.org/10.4000/ encyclopedieberbere. 2713

Ce document a été généré automatiquement le 17 février 2022.

(c) Tous droits réservés 


\title{
Nemencha : Société et Langue
}

\author{
Malek Boudjellal
}

1 Le terme Nemencha, parfois transcrit Nementchas ${ }^{1}$, désigne à la fois un sous-ensemble géographique de l'atlas saharien et une confédération tribale berbère chaouïa.

\section{Le pays nemouchi}

2 A l'est de l'imposant massif de l'Aurès, coule la rivière Mellagou (Oued el-Arab) qui est la frontière naturelle avec les monts des Nemencha. Cet espace se présente comme un ensemble de montagnes dont les plis sont plus lâches, le relief plus tassé quoique les sommets du Bou Djellal et du djebel Dokhana ne manquent pas de faire impression, ce dernier culminant à $1.712 \mathrm{~m}$. Enfin le djebel Foua, qui atteint presque les $1.500 \mathrm{~m}$ et le Djebel Onk $1.338 \mathrm{~m}$ ne sont pas dépourvu de grandeur. Au sud, des couches calcaires faiblement ondulées forment un plateau qui plonge rapidement sous les alluvions du Sahara. Les monts des Nemencha sont creusés de gorges profondes et présentent localement un paysage de causses ${ }^{2}$.

3 La zone montagneuse de climat tempéré où les valeurs du thermomètre ne dépasse guère $36^{\circ}$ à l'ombre dans les journées les plus chaudes, mais il descend assez fréquemment à -4 et $-5^{\circ}$ en hiver; aussi le djebel Chéchar et les monts environnants gardent-ils, pendant quelques mois leurs cimes enneigées.

4 Le pays Nemencha est peu boisé, les pentes pierreuses du Chéchar et en particulier celles ravinées du Mahmel demeurent désespérément stériles. Pays de steppe recevant en moyenne moins de $450 \mathrm{~mm}$ de pluies annuelle, la végétation y est réduite à de maigres genévriers parsemant les crêtes sèches, tandis que l'alfa y triomphe.

5 Par contre le reste des Monts des Nemencha, le Djebel Onk, les djebel Bou Djellal et Dokhana conservent des pâturages jusqu'en été et permettent l'élevage intensif du mouton.

6 Le territoire des Nemencha s'étend de Khenchela à Tébessa d'ouest en est (environ $150 \mathrm{Km}$ ), du nord du Djebel Chettaya à la région des Chotts, dans le sens nord-sud. Ainsi, les Nemencha sont limités à l'Ouest par les tribus chaouïa du Djebel Chéchar ${ }^{3}$ (Ah 
Barbar et Ah Maafa) et les Amamra, au nord par la confédération tribale chaouïa des Harakta $^{4}$ et la tribu arabophone mara-boutique des Ouled Sidi Yahia, à l'est par une autre tribu arabophone mara-boutique ${ }^{5}$ les Ouled Sidi Abid qui s'étendent le long de la frontière tunisienne, au sud, ils sont limitrophes des tribus sahariennes arabophones du Souf.

Le territoire des Nemencha se subdivise en deux parties :

- La première située sur les Hauts plateaux, où se trouvent presque tous les terrains de cultures de la tribu, constitués par les plaines tel celles de Sbikha et Garet.

- La seconde plus vaste comprenant les monts des Nemencha et le piémont saharien, qui constitue les terrains de parcours et d'épandage des crues.

8 La confédération des Nemencha est certainement une des plus grande en nombre d'individus $^{6}$ et la plus importante par rapport à l'étendue territoriale des confédérations tribales berbères Chaouïa. Son nom berbère est $A h^{7} N e m m u s^{8}$.

D'après L. Golvin (p. 222) : « quelque soit la tribu ou fraction de tribu qu on questionne tous les habitant de la grande confédération des Nemencha se disent arabes et voient leur lointaine origine dans les envahisseurs beni Soleym ».

De même, selon A. Vaissiere: «Bien que vraisemblablement d'origine berbère et que leur langue usuelle soit le chaouïa, les mythes fondateurs de la tribu donne pour ancêtre éponyme un certain Rechaïch ben Ouchah Ben Mohammed ben Ahmed Ben Uthman, un arabe hilalien qui s'était installé et avait fait souche dans la région de Chechar" (Revue Africaine 1892, p. 236-237).

11 Cependant, comme les deux auteurs l'ont constaté, le seul idiome utilisé par les femmes et hommes ayant encore l'usage de la tente et des maisons de terre était le chaouïa ; d'où l'on peut conclure qu'il serait assez risqué d'accorder une foi entière à ces traditions locales sachant que jusqu'à une date récente il était de bon goût de se prévaloir d'une origine arabe en Afrique du Nord; les résultats d'une enquête actuelle seraient sans aucun doute très différents. Qu'il y ait eut parmi la confédération des Nemencha l'absorption d'éléments hilaliens et soleymites ne fait cependant pas de doute, d'ailleurs certains récits n'accordent des origines arabes hilaliennes qu'à un nombre restreint de familles.

12 L'imprégnation de la littérature orale nemouchie d'éléments de la geste hilalienne (la taghrîba $a^{9}$ pourrait conforter l'hypothèse de la berbérisation d'éléments arabes, accompagnée de la transmission d'une partie de leur patrimoine oral, à l'instar de ce qui s'est passé chez les Aït Waraïn de l'aire du tamazight (Moyen Atlas) avec les BeniChkoud $^{10}$.

13 Néanmoins, il est un fait établi que le berbère résiste mieux chez les Nemencha de la wilaya de Khenchela (Ah Rechaïch) alors que dans la wilaya de Tébessa l'arabisation y est plus profonde et plus ancienne. Ainsi dans l'enquête de 1913 d'Edmond Doutté et Emile-Félix Gautier, des fractions importantes des Brarcha et Allaouna sont décrites comme étant arabo-phone exclusive :

«Dans les sept autres fractions des Allaouna, les indigènes parlent chaouïa entre eux, dans leurs familles et au dehors, lorsqu'ils ne veulent pas être compris de ceux qui les environnent. Ils convient d'ajouter que même dans les fractions des Allaouna et Brarcha, où le chaouïa a subsisté, la presque totalité des hommes et la majorité des femmes, les jeunes surtout, savent assez d'arabe pour s'exprimer et se faire très suffisamment comprendre dans cette dernière langue.

Aux Brarcha, huit fractions sur seize parlent arabe de temps immémorial ; ce sont les Ouled- 
Khelifa-Dehara, Ouled-Khelifa-Gueballa, Ouled-Chenina, Ouled Belharrets, Ouled-Saïdane, Ouled Si Ali Ouled Saci et Djeurf. Une autre fraction, celle des Ouled Chekor, a adopté l'arabe il y a une quarantaine d'années (p. 75 « Le berbère dans le cercle de Tébessa »). les Brarcha et les Ah Rechaïch, qui elles-mêmes se subdivisent selon le tableau suivant:

\begin{tabular}{|c|c|c|}
\hline \multirow[t]{2}{*}{ I. Allaouna } & \multirow[t]{2}{*}{ I.1. Ijlenden } & Boucoussa \\
\hline & & Harrat \\
\hline & & Moussa \\
\hline & & Tkaka \\
\hline & & Djouj \\
\hline & & Lamra \\
\hline & I.2. Ah Briq & Zghema \\
\hline & & Chamekh \\
\hline & & Aounallah \\
\hline & & Aissaoui \\
\hline & & Saad \\
\hline II. Brarcha & II.1. Izghemmen & Ah Amar \\
\hline & & Ah Mbarek \\
\hline & & Ah Slimane \\
\hline & & Ah Trahna \\
\hline & II.2. Ah Mahboub & Ah Chekour \\
\hline & & Ah Djilal \\
\hline & & Ah Bouyahia \\
\hline & & Ah Brahim \\
\hline & II. 3. Les Ah U Seit : & Ah Messaoud \\
\hline
\end{tabular}




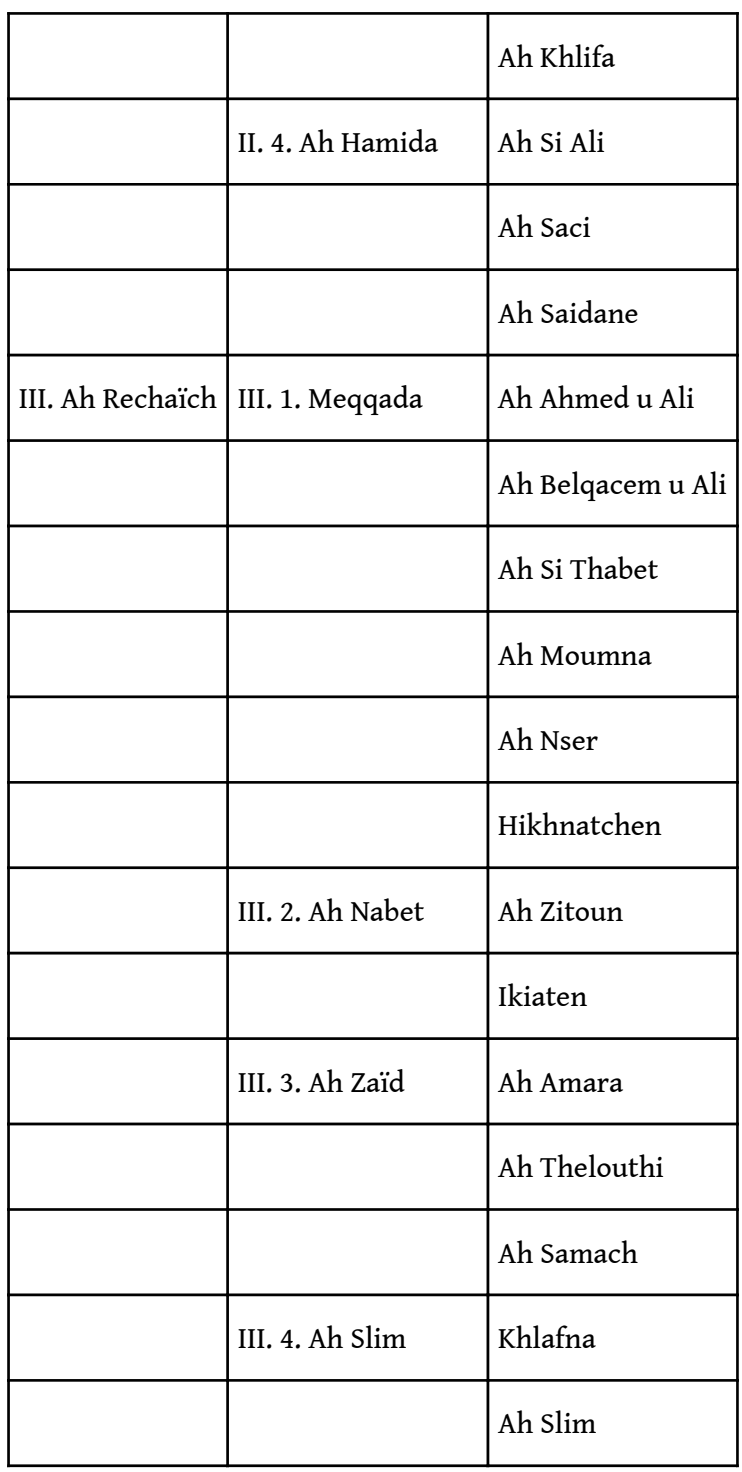

\section{Le mode de vie}

16 Les Nemencha était à l'exemple des autres tribus chaouïa de l'Aurès oriental et méridional y compris vers les hautes plaines constantinoises des nomades pasteurs habitant la tente (axxam ou aqidun), qu'ils ont progressivement abandonné au profit d'un habitat en dur (terre crue, pierres et tuiles) consacrant leur sédentarisation pendant le $\mathrm{XX}^{\mathrm{e}}$ siècle. Ce mouvement de fixation s'est accéléré pendant la guerre d'Algérie, par la création de certains villages de regroupement ${ }^{12}$ comme ce fut le cas de Tazougaght (wilaya de Khenchela), ce qui n'empêche pas le maintien d'un habitat dispersé (aquwwar). 


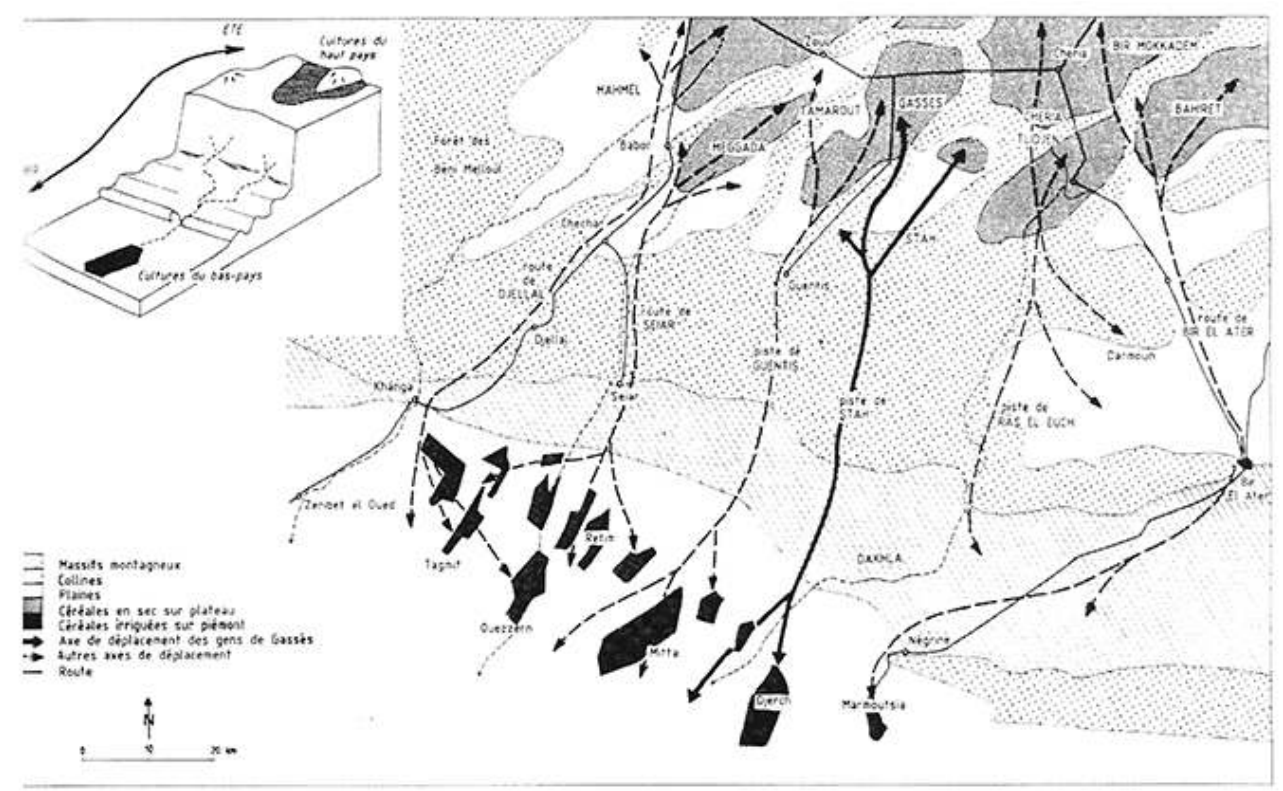

Fig. 29. Le pays Nemẹmcha et les axes de déplacement.

Au nord, le haut pays, à 1200 mètres d'altitude, lieu de vie d'été. Au sud, le piémont, à 100-200 mètres d'altitude, lieu de vie d'hiver. Les axes de déplacement qui relient haut et bas pays fonctionnent pour les cultures et pour l'élevage. Les déplacements se faisaient traditionnellement à pied. ils se font souvent en camion aujourd hui.

Fig. 1. CARTE des dÉPLACEMENTS dANS Les Nemencha. d'APRÈs M. Côte 1996, P. 55.

En pays steppique, la recherche de complémentarités régit l'économie des communautés. Dans cette région, le relief peut descendre facilement, sur une centaine de kilomètres, de 1.200 à $100 \mathrm{~m}$ d'altitude, et la pluviométrie baisser de 450 à $150 \mathrm{~mm}$. Ceci implique que l'on effectue des déplacements :

- Pour les cultures : «à la céréaliculture précoce sur épandage de crue pratiquée sur le piémont saharien succède la céréaliculture tardive pratiquée par les mêmes groupes sur les hautes terres. » (Côte 1979, p. 52).

- Pour le cheptel ovin qui ne peut être nourri par la steppe que durant une courte période (trois à quatre mois). Aux autres périodes, la compensation du manque de nourriture est assurée grâce au déplacement des hommes et des bêtes en des lieux différents tout au long de l'année.

Cette société est encore marquée par le mode de vie agro-pastoral, bien qu'il ait subi des mutations considérables, dont les aspects les plus visibles sont :

- Le déplacement du cheptel se fait par camion;

- La culture des céréales des piémonts a fait place aux fourrages ;

- La généralisation de l'enseignement a fixé dans les centres urbains les familles dont seuls quelques membres se consacrent encore à la transhumance.

Au plan global, des changements considérables ont affecté profondément la société :

- A partir des années 1950, elle est devenue un foyer pourvoyeur d'émigration vers la France ;

- Elle a connu un exode rural interne à l'Algérie important vers les deux grandes villes de la région, Khenchela et Tebessa, voire bien plus loin jusqu'aux villes du littoral Annaba, Skikda et Alger.

De leur ancienne vie semi-nomade les Nemencha ont conservé un artisanat lié au pastoralisme, à savoir le tissage sous forme de tapis de haute laine nouée (metrah), laine bouclée (aqdif, tarakna, tazerbit), et des tapis plus fins et ras (ddragt) servant jadis à 
cloisonner les espaces sous la tente; on les retrouve de nos jours meublant les salons des intérieurs.

A l'inverse du massif de l'Aurès où le travail de la laine est exclusivement féminin, chez les Nemencha, les hommes sont impliqués dans des tâches plus complexes pour la réalisation des ornements, toute confection de tapis requérait le savoir du reggam ${ }^{13}$. Actuellement, cette corporation voit son nombre décroître en raison de l'âge avancé des détenteurs de ce savoir-faire sans qu'il y ait de relève par la jeune génération masculine ; cependant les femmes de la tribu récupèrent à leur compte les techniques de composition des décors ${ }^{14}$. L'excellent travail de Lucien Golvin autour des arts traditionnels algériens confirme cette tendance dès 1953. Les centres de production du tapis des Nemencha sont situés actuellement dans les villes de Khenchela, Babar et Tebessa.

Le sentiment d'appartenance tribale est encore bien vivant. Il réapparaît à chaque élection locale par opposition aux tribus voisines, les Amamra au niveau de la wilaya de Khenchela, les Ouled Sidi Yahia et Ouled Sidi Abid au niveau de la wilaya de Tebessa, ou lors du partage des terres sahariennes qui oppose la collectivité nemouchie à l'Etat central.

Sur le plan culturel la population est restée en majorité fidèle à la langue berbère et aux productions culturelles traditionnelles comme le chant et la musique chaouï du style arehbi (polyphonie) et rokroki (flûte et bendir).

Le pays nemouchi, comme la grande majorité de la zone chaouïa, a une élite de formation plutôt arabisante, mais ceci n’a pas empêché, depuis les années 1980, une agitation et production culturaliste berbère. Pour la production poétique moderne, on citera Bachir Adjroud, Omar Bouziane, et Tayeb Djellal. On relève aussi la production de textes en berbère (contes, recueil de chants) ou sur le berbère, certains ayant été édités d'autre restant à l'état de manuscrits inédits ; parmi ces producteurs, on citera Tayeb Djellal, Sahraoui Djamila, Nemelli Abdallah, et Hadjadj Madani. Enfin aux niveaux des médias d'expression chaouïa, on constate que plusieurs animateurs radio et télévision (chaîne II nationale, chaîne 4 d'expression amazighe en Algérie, et Radio Khenchela) sont originaires des Nemencha.

\section{La langue (notes linguistiques)}

Le parler des Nemencha représente l'extension la plus à l'est des parlers chaouïa*. Il est en contact direct avec les parlers arabes bédouins ${ }^{15}$. Il partage avec l'ensemble des parlers chaouïa certains grands traits :

- La spirantisation* d'une partie des occlusives simples berbères $/ d />[d], / d+>[d]$ et $/ t />[t]$ et partiellement de la palatale $/ \mathrm{k} />[\mathrm{k}]$ qui $\mathrm{n}$ affecte pas tout la zone nemouchie ${ }^{16}$.

- Le traitement particulier des palatales $/ \mathrm{k} />/ \check{s} /$, et $/ \mathrm{g} />/ \check{z} /$.

- La chute de la voyelle initiale dans les lexèmes nominaux de type vCvC.

- La négation discontinue de type ur $(>u d)$... $\check{s}(a)$.

Le parler nemouchi fait partie du groupe oriental du chaouï ${ }^{17}$ dont les traits linguistiques les plus marquants sont:

- L'affaiblissement systématique $\mathrm{du} / \mathrm{t} / \mathrm{>}>\mathrm{h}>\varnothing:$ (h)ametțut «femme », (h)addert " maison ", (h)ametna « pluie »;

- L'usage du pronom régime direct et indirect de la $2^{\mathrm{e}}$ personne du pluriel en -kum / $k u m t^{18}$; 
Dans cet ensemble oriental, le parler nemouchi a ses propres caractéristiques phonétiques, morphologiques et lexicales qui lui confèrent une physionomie particulière reconnaissable de suite par une oreille avertie.

\section{Particularités phonétiques et morphologiques}

28 - Tendance très nette à la disparition régulière de la vélaire $/ \mathrm{\gamma} /$ en position finale avec remplacement par un /a/ allongé, notamment pour l'indice de la $1^{\text {ère }}$ personne du singulier pour certain verbes et les affixes directs et indirects des verbes, des prépositions et du nom (possessif) de la $1^{\text {ère }}$ personne du pluriel ${ }^{19}$ :

ttett $\bar{a}<$ ttette $\gamma$, «je mange »

uggurā < ugire $\gamma$, "j'ai marché »

yuš-anā <yuš-ane $\gamma$, « il nous a donné »

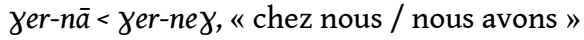

29 - L'usage d'un pronom régime direct et indirect de la $1^{\text {ère }}$ personne avec un/i/comme voyelle initiale : iyi/iya

ušin-iyi-dd / ušin-ay-dd, « ils m'ont donné »

yenn-iya / yenn-aya, « il m'a dit »

30 - L'usage des pronoms autonomes $2^{\mathrm{e}}$ personne masculin et féminin : kenwi/ kennemti, le reste des parlers du groupe oriental a kummim/kumentin -Les assimilations :

$n+w>w w$; exemple: fus wwergaz < fus $n$ wergaz, « la main de l'homme »

31 - La désinence personnelle de l'impératif masculin pluriel a la forme X-m et non X-t.

\section{Quelques particularités lexicales}

C'est par certaine réalisation phonétique, morphologique, mais surtout lexicale que l'on reconnaît un locuteur originaire des Nemencha parmi l'ensemble chaouïa. Ces lexèmes peuvent être aussi bien des mots berbères ou des emprunts à l'arabe, en usage uniquement chez eux, ou que l'on retrouve dans d'autres dialectes berbères hors $d u$ domaine chaoui.

Le tableau ci-dessous illustre la spécificité lexicale du parler des Nemencha par rapport au parler des Harakta, leurs voisins du nord.

\begin{tabular}{|l|l|l|}
\hline Parler des Némencha & Parler des Harakta & Français \\
\hline ałegg $^{\circ} a l$ & aberkan & Noir \\
\hline azellum & abeggas & Ceinture \\
\hline atrar & ajdid & Neuf \\
\hline azen & serf & Envoyer \\
\hline ska & bna & Construire, monter une tente \\
\hline sgujder & snezgem & s'inquiéter, causer du souci \\
\hline
\end{tabular}




\begin{tabular}{|l|l|l|}
\hline agejdur & anezgum & Soucis \\
\hline kan & zir/berk & Seulement \\
\hline ameččuk & ahu & Un garçon \\
\hline xirella & labas & Beaucoup \\
\hline ugur & ugir & Marcher \\
\hline tanezzayt & tașebhit & Matinée \\
\hline sqed & idḍu & Tomber \\
\hline ful & ibawen & Fèves \\
\hline ttefaḥ & adeffu & Pomme \\
\hline ahmam & adbir & Ramier \\
\hline luqq-a & imir-a ${ }^{20}$ & Maintenant \\
\hline amezžur & amejju & Oreille \\
\hline ayečča & adečča & Demain \\
\hline zewwez ${ }^{21}$ & mucel & Olives \\
\hline Lget & azemmur & \\
\hline zzitun & & \\
\hline
\end{tabular}




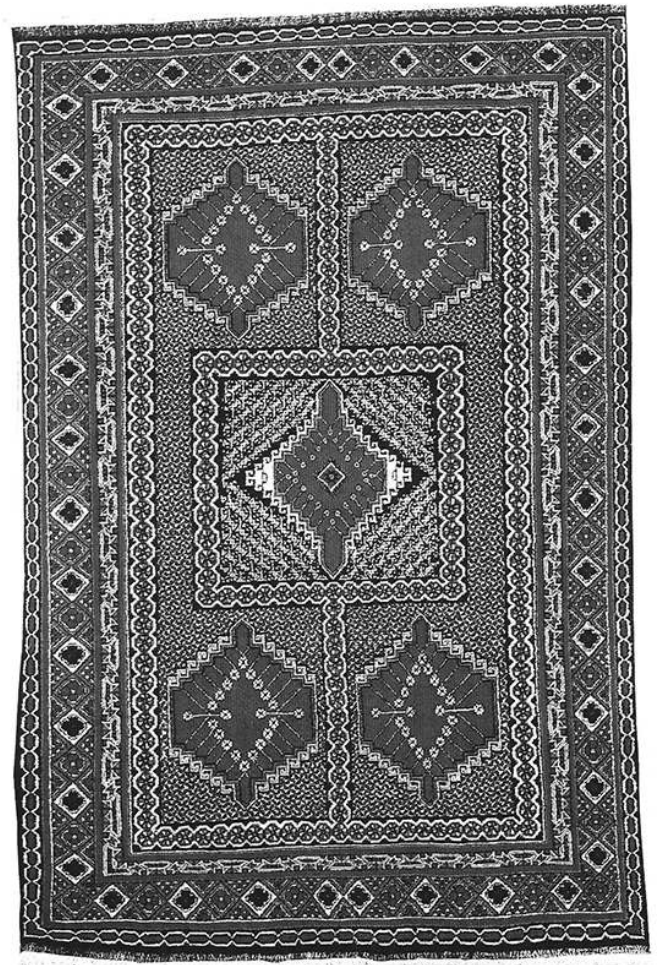

Fig. 2. TAPIS DES NEMENCHA.

\section{BIBLIOGRAPHIE}

BOUDJELLAL M., 2007 - Esquisse de description du parler chaouïa des Nemencha de Tazougaght, Maitrise, Paris, INALCO.

BOUdJELLAL M., 2008 - Contribution à la géographie linguistique du berbère chaouïa, Master II, Paris, INALCO.

CHAKER S., 1986 - « Aurès » (Linguistique), Encyclopédie berbère VIII, Edisud.

COTE M., 1996 - Pays, paysages et paysans d'Algérie, CNRS Editions, Paris

DESPOIS J., 1942 - « La bordure saharienne de l'Algérie orientale » in Revue africaine DESTAING. E., 1920 - Etude sur le dialecte berbère des Aït Seghrouchen (Moyen Atlas marocain), Paris, Edition Ernest Leroux

DIELEMAN F., 1994 - Esquisse de description de la langue berbère chaouïa - variations lexicales et phonétiques et investigation sociolinguistique, Maîtrise, Université de Provence.

DJELLAL T., 2005 - Si tinfusin umadal (contes du monde), Alger, HCA.

DOUTTÉ E. et GAUTIER E. F., 1913 - Enquête sur la dispersion de la langue berbère en Algérie, Alger. 
ESTABLET C., 1987/3 - « Administration et tribu chez les Némemcha (Algérie) au XIX ${ }^{\mathrm{e}}$ siècle », in ROMM 45.

FELLAH Dj., 2009 - Chants des Aurès (Ahazidj min al Awras), Alger, Edition Chihab (en arabe).

FERAUD Ch., 1868 - « Kitâb-el-Adouânî ou le Sahara de Constantine et Tunis », Recueil des notices et mémoires de la Société archéologique de la province de Constantine, vol. 12/1, p. 1-208.

GOLVIN L., 1953 - Les arts populaires d'Algérie, t. II, Les tapis algériens, Alger, Typo-Litho et J.

Carbonel.

LARTIGUE Colonel R. de, 1904 - Monographie de l'Aurès, Constantine, Marlé-Andrino.

MARCAIS Ph., 1957 - « Les parlers arabes » in Initiation à l'Algérie, (p. 215-237)

MASQUERAY E., 1876 - « Voyage dans l'Aourâs », Bulletin de la Société de Géographie, juillet (texte, p. 55-56).

MASQUERAY E., 1879 - Comparaison d'un vocabulaire du dialecte des Zénaga avec les vocabulaires correspondants des dialectes Chaouia et des Beni Mzab, Paris, Imprimerie Nationale, (Archives des missions scientifiques, série III, t. 5), p. 473-533.

MASQUERAY E., 1878 - « Le Djebel Chechar », Revue africaine, 22, p. 26-48, 129-145, 202-214, 259-281.

MASQUERAY E., 1885 - « Traditions de l'Aourâs oriental », Bulletin de Correspondance Africaine, III, p. $72-110$.

MORIZOT P., 1989 - L'Aurès ou le mythe de la montagne rebelle, Paris, L'Harmattan.

SCANNAVINO Ch., 1957 - « La tente chez les nomades des Nemencha (département de

Constantine) ", Cahier des techniques de l'Afrique du Nord, n 4, p. 41-45.

VAISSIÈRRE A., 1892 - « Les Ouled-Rechaich », Revue africaine, p. 208-243.

VAISSIÈRRE. A., 1893 - « Les Ouled-Rechaich » (suite), in Revue africaine, p. 5-21 et 136-150.

\section{NOTES}

1. La prononciation locale est nnemamša, pluriel du gentilé nemuši (prononcé aussi lemuši); dans les documents officiels algériens l'orthographe utilisé est Namamcha.

2. Terme occitan usité en géographie pour désigner les parties sommitales et les versants des montagnes ayant des étendues xériques peu épaisses et dont la roche affleure parfois.

3. Il est à noter que le Djebel Chéchar est la partie occidentale des Monts des Némencha, d'apres certaines traditions locales et les sources écrites (Kitâb el-Adouânî, Musqueray), les Némencha en aurait été chassé par les Béni Barbar, au courant du moyen-âge.

4. Côte M., EB XXII, « Haracta », p 3392-3394.

5. Voir pour les Ouled Sidi Abid: Murati P., «Le maraboutisme ou la naissance d'une famille éthnique dans la région de Tebessa », Revue africaine 80, 1937, p : 256-315.

6. Golvin Lucien les estimait à 120.000 en 1956, d'après le découpage actuel des wilayas en Algérie on pourrait les estimer à environ 400000 .

7. Ah est la prononciation locale du Aï (fils de X) pan-berbère.

8. Nemmuš l'étymologie populaire décompose le mot en $n+$ Mošé : celui de Moshé ou fils de Moïse de là a donner une origine juive ou judaïsante à cette confédération tribale, n'oublions pas que nous sommes dans le «pays de la Kahina »! (Sources personnelles recueillies oralement dans la région de Khenchela). 
9. Taghrîba . étymologiquement « le départ vers l'ouest » récit épique des Banu Hillal. Ceux-ci ont conservé l'histoire de leur épopée dans une série de récits mi-historiques, mi-légendaires appelés « geste hilalienne » que l'on retrouve dispersés du Nedjd au Maroc.

10. Peyron M., « Chants », EB XII, p 1866, 1867.

11. La quatrième composante des Némencha est la tribu des Ah Khiar. Cette dernière a migré vers la région de Souk-Ahras entre le XVI ${ }^{\mathrm{e}}$ le XVII ${ }^{\mathrm{e}}$ siècle (d'après une source orale recueillie par moimême, confirmée par le Kitâb El-Adouânî).

12. Village créé pendant la guerre d'Algérie pour regrouper la population éparpillée dans les douars afin de mieux la contrôler et priver de base arrière le FLN.

13. Voir l'Article « Le Reggam » de Golvin », Annales de l'Institut d'Etudes Orientales (Alger), 9, 1951.

14. Le même phénomène s'est produit au Djebel Amour voir EB IV, p. 604.

15. Voir Ph. Marçais, 1957 - « Les parlers arabes » in Initiation à l'Algérie, (p. 215-237).

16. Dans d'autres parlers qui correspondent à des ensembles tribaux, cette spirantisation est généralisée, c'est le cas par exemple chez les Aït Daoud.

17. Ce groupe oriental rassemble les parlers des tribus voisines: Harakta, Amamra, tribus du Chéchar. Les deux autres groupes de parlers chaouïa sont les parlers centraux (massif de l'Aurès) et les parlers occidentaux (Monts du Bélezma).

18. Ce trait constitue une ligne d'isoglosse nette opposant le groupe oriental aux deux groupes central et occidental. Ce même trait se retrouve dans le parler berbère de Ouargla (Sahara algérien) et certains parlers du Rif marocain.

19. Même phénomène observé dans les parlers de Ouargla, et de Tattaouine (sud tunisien).

20. Il n'est pas inconnu du parler nemouchi, mais il est utilisé dans le passé ou dans le futur pour désigner une action pas très lointaine.

21. Le verbe rcel n'est utilisé que chez certaines fractions des Némencha de Tebessa (Troubla).

INDEX

Mots-clés : Aurès, Economie, Ethnologie, Géographie, Linguistique 